\title{
EL MODELO GAVILÁN Y LA COMPETENCIA EN MANEJO DE INFORMACIÓN EN UNIVERSIDAD DE LAS FUERZAS ARMADAS ESPE
}

\section{GAVILAN MODEL AND INFORMATION MANAGEMENT COMPETENCE AT THE UNIVERSIDAD DE LAS FUERZAS ARMADAS ESPE}

\author{
Ing. Fabián Jaramillo Campaña Mgs, Dra. Aída Bedón Bedón Ph.D. \\ Universidad de las Fuerzas Armadas ESPE \\ Sangolquí, Ecuador - Av. General Rumiñahui s/n \\ fajaramillo@espe.edu.ec, anbedon@espe.edu.ec
}

\section{Resumen}

Proceso investigativo de varios años (2010-2014). Inicia con diagnóstico de competencia en manejo de información (CMI), estudiantes de la Universidad de las Fuerzas Armadas ESPE de Ecuador (2010). Se identificó que poseían, en promedio, el $28,38 \%$ de $\mathrm{CMI}$, debido a inexistencia de un proceso eficiente de búsqueda y procesamiento de la información.

Hipótesis: "el uso sistemático de un modelo de solución de problemas de información, Modelo Gavilán, elevaría la CMI en los mismos". Se emprende con un estudio cuasi-experimental, se toman grupos de estudiantes que ya están integrados en Cursos, las unidades de análisis no se asignan al azar. Se usa el diseño del pre-test y pos-test.

Se aplicó el Modelo Gavilán, durante dos semestres académicos (20132014), en asignaturas Metodología de Investigación y Proyecto Integrador II. Este estudio se realizó con 112 estudiantes: ingenierías técnicas (42), ciencias administrativas (32) y educación infantil (38).

Los resultados evidenciaron un incremento significativo de la $\mathrm{CMI}$, en todos los grupos participantes: empezaron con 38,54\% (Pre-test - promedio CMI), en seis semanas de entrenamiento con dicho modelo, alcanzaron $70,39 \%$; para concluir el semestre con $80,87 \%$. En cinco meses se incrementó en $109,82 \%$. Se duplicó el nivel inicial de la CMI de los estudiantes.

Palabras clave: Modelo Gavilán, competencia en manejo de información, alfabetización informacional, educación superior, Ecuador.

\footnotetext{
Abstract

It is a research process of several years (2010-2014). It starts with a diagnosis about information management competence (IMC), applied in a group of students at Universidad de las Fuerzas Armadas ESPE - Ecuador (2010). They
} 
were found to have an average of $28.38 \%$ of IMC, due to lack of an efficient search process and information processing.

Hypothesis: "the systematic use of a model of troubleshooting information, Gavilan Model, would raise the level of IMC in them." It is undertaken with a quasiexperimental study. Groups of students who have already been integrated into courses. The units of analysis are not assigned randomly. The design of pre-test and post-test is used.

The Gavilan Model was applied in two semesters (2013-2014), with subjects Research Methodology and Integrative Project II. This study was conducted with 112 students of engineering (42), business administration (32) and education (38).

The results showed a significant increase in the CIM in all participating groups, they began with $38.54 \%$ (Pre-test - average IMC), in six weeks of training with this model, reached $70.39 \%$, and the semester finished with $80.87 \%$. In five months, it increased a $109.82 \%$; so, the initial level of the IMC of students was doubled.

Keywords: The Gavilan Model, information management competence, information literacy, higher education, Ecuador.

\section{Introducción}

La Era digital, inaugurada con el desarrollo de la micro-electrónica y el aparecimiento del computador digital, ha producido un vertiginoso y constante desarrollo de las nuevas tecnologías de información y comunicación (NTIC), de la multimedia (texto, imágenes, sonido, video y animación), de la telemática y la Internet, han provocado un verdadero "Cambio de Época" (De Souza, 2014), desde hace varias décadas, hecho que provoca la transición de la Sociedad Industrial a la Sociedad de la Información y el Conocimiento, y más aún, hacia la Sociedad Red y las tecnologías disruptivas.

De acuerdo con Garmendia (2005), la alfabetización informacional (information literacy) o competencia en manejo de información, como lo señala Cacheiro (2014), "es un término que se ha utilizado para definir el todo del ciclo de la información que va desde la búsqueda de datos, pasando por el uso de la información y finalizando con la generación y distribución de conocimientos". Coincidimos plenamente con la misma autora cuando señala que la CMI "implica no solo la búsqueda de información, sino una búsqueda organizada que facilite la selección minuciosa y el análisis profundo de ésta para la generación sistemática de nuevos conocimientos".

Por otro lado, la CMI tiene relación con el desarrollo de competencias de gestión de la información, lo cual incluye identificar el medio que responde a la necesidad, una selección bien fundamentada de fuentes y una evaluación que conduce al buen uso de la información para sí mismo y para la sociedad (Azinian, 2009).

En los últimos tiempos, se ha incrementado el interés por la competencia en el manejo de la información, pues la misma está relacionada con el aprendizaje continuo y el aprendizaje para toda la vida, propiciando un desempeño profesional 
El Modelo Gavilán y la Competencia en manejo de información en la Universidad de las Fuerzas Armadas ESPE

y personal exitoso. (Sánchez \& Alfonso, 2007). De igual manera, la CMI está relacionada con el aprender a aprender, como lo señala Carratalá (2013).

La competencia en manejo de información es vital para la vida social, individual y colectiva (Galindo, 1996), ella representa una competencia vital para las personas, profesionales, instituciones y sociedad, impactando en los campos social, económico, cultural, tecnológico y científico, en este sentido representa el cimiento de dos actividades de enorme importancia: el aprendizaje y la investigación científica. Otros autores consideran a la competencia en el manejo de la información como una parte de la competencia digital (Vivancos, 2008), (Moya \& Luengo, 2011).

No hay certeza alguna de que Internet proporcione un conocimiento seguro, si los usuarios no adoptan un modo de operar adecuado para ello (Colle, 2003), esto es, si no desarrollan competencia en manejo de información (CMI).

Desde hace varias décadas, para estructurar los procesos de búsqueda y procesamiento de información, para hacerlo más eficiente, existen los modelos de solución de problemas de información, entre ellos el Modelo Gavilán, el mismo que se ha adaptado a estándares de la alfabetización informacional (ALFIN), fortaleciendo determinados aspectos a diferencia de otros modelos (GonzálezFernández-Villavicencio, 2014).

Cabe señalar que los procesos de investigación formativa y la formación de investigadores, coincidiendo con Juárez-López y López-Pérez (2012), se dan a partir del abordaje de problemas científicos, que sin duda, "requiere de estrategias lógicas que permitan el acceso y procesamiento de información”. En este sentido, el Estatuto de la Universidad de las Fuerzas Armadas ESPE, en el Artículo 2 señala como Misión: “... formar académicos, profesionales e investigadores de excelencia, creativos, humanistas, con capacidad de liderazgo, pensamiento crítico y alta conciencia ciudadana; generar, aplicar y transferir el conocimiento; y, proporcionar e implementar alternativas de solución a los problemas del país, acordes con el Plan Nacional de Desarrollo; siendo su Visión permanente ser una universidad líder en la gestión del conocimiento y tecnología en el sistema de educación superior en el país, con prestigio internacional" (ESPE, 2015).

El Modelo Gavilán fue creado por la Fundación colombiana Gabriel Piedrahita Uribe y es difundido por el Portal educativo Eduteka ${ }^{1}$. y consta de los siguientes pasos:

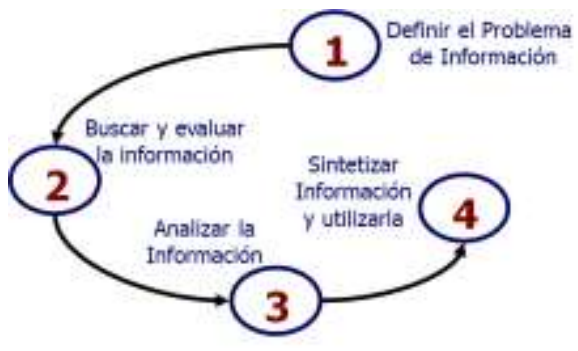

Figura 1 Pasos del Modelo Gavilán (Fundación Gabriel Piedrahita Uribe, Eduteka, 2007).

${ }^{1}$ www.eduteka.org 


\section{Método}

Para esta investigación, se plantea un estudio cuasi-experimental, porque se toman los grupos de estudiantes que ya están integrados, por lo que las unidades de análisis no se asignan al azar. Se usa el diseño del pre-test y postest, respecto al uso sistemático de un modelo de solución de problemas de información, en este caso, el Modelo Gavilán durante el proceso de aprendizaje en las asignaturas Metodología de la Investigación y Proyecto Integrador II. Durante los semestres agosto 2013 - enero 2014 y marzo 2014 - agosto 2014. Este estudio se realizó con 112 estudiantes, distribuidos de la siguiente manera: ingenierías (42), ciencias administrativas (32) y educación infantil (38), cuyo detalle se presenta en las Figuras 2 y 3.

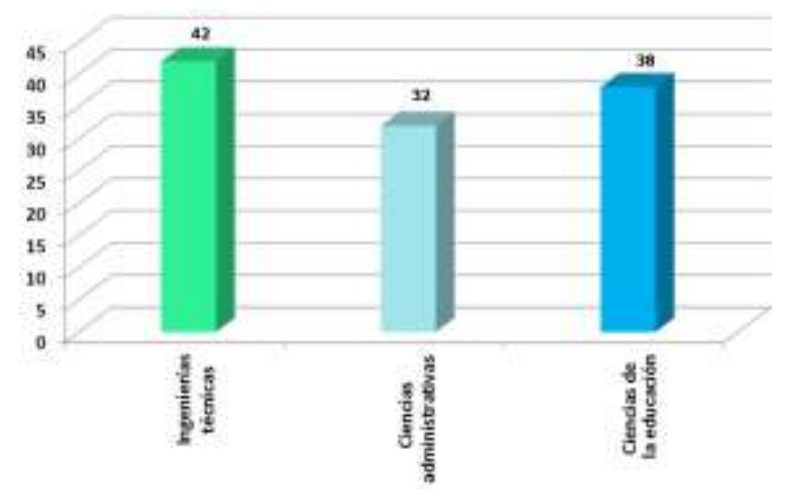

Figura 2 Población estudiantil por áreas de especialización 2013-2014

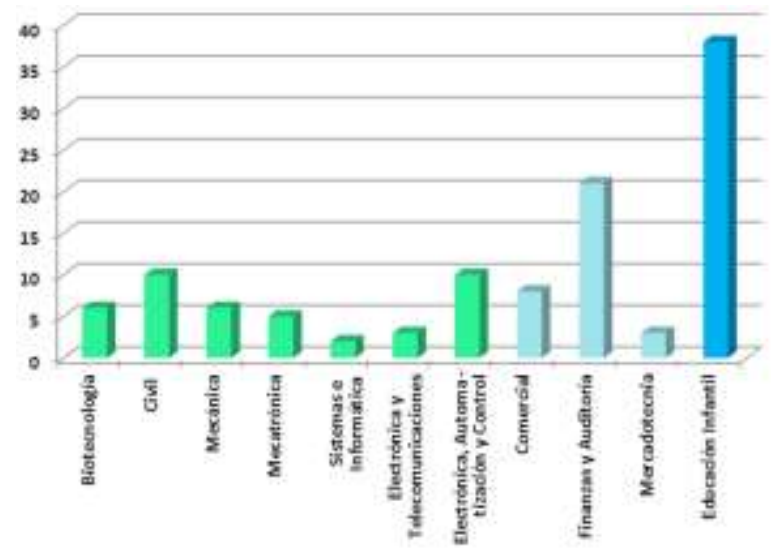

Figura 3 Población estudiantil por Carreras

Este estudio cuasi-experimental, se concentro en identificar la relación existente entre la aplicación del Modelo Gavilán y el incremento de la CMI estudiantil, para lo cual se tomaron mediciones en tres momentos del proceso: 
El Modelo Gavilán y la Competencia en manejo de información en la Universidad de las Fuerzas Armadas ESPE

pre-test (inicio), post-test 1 (seis semanas de entrenamiento con el Modelo Gavilán) y post-test final (5 meses del proceso formativo total, para cada grupo).

Se aplicó encuestas para recoger la información, en los tres momentos del experimento y se identificó los componentes de la Competencia en manejo de la información. El instrumento se fundamenta en 13 dimensiones y 67 indicadores de esta variable.

Se realizó el análisis estadístico utilizando el programa Excel y la prueba t de Student para probar la hipótesis. Las hipótesis consideradas fueron las siguientes:

Ho1 : $\mu$ pre-test $-\mu$ post-test final $=0$ Ho2 : $\mu$ pre-test $\geq \mu$ post-test final

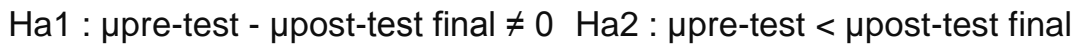

De manera puntual, el trabajo con los estudiantes consistió en aplicar todos los pasos con sus subpasos del Modelo Gavilán, que son los siguientes:
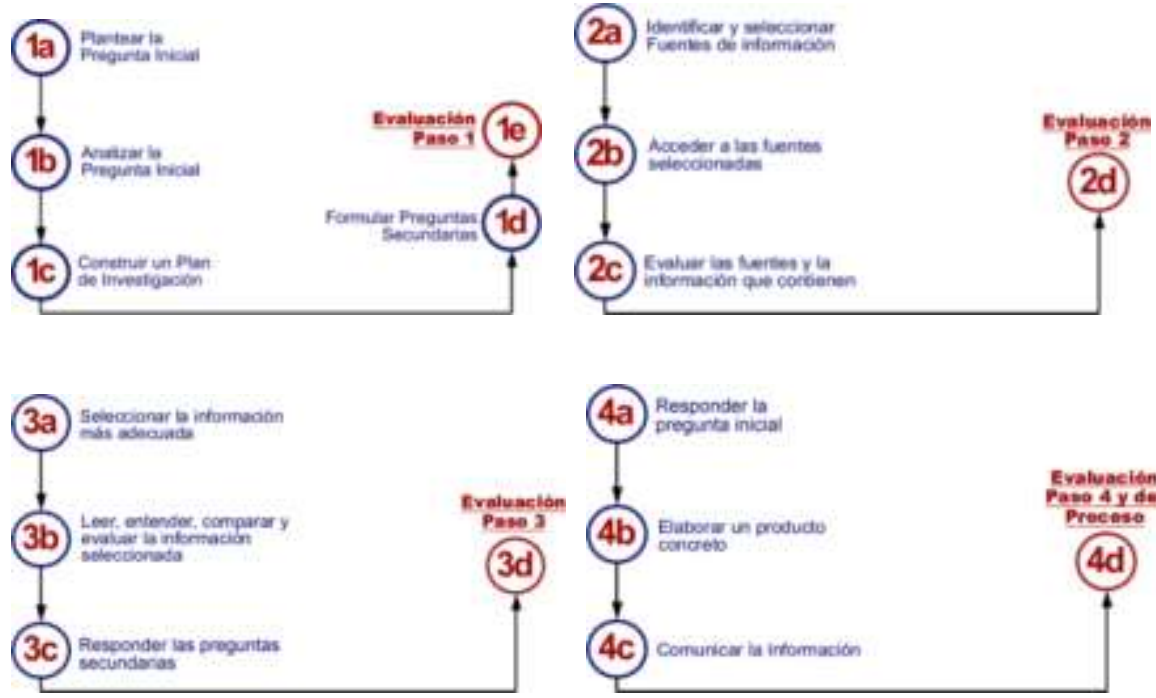

Figura 4 Sub-pasos Modelo Gavilán (Fundación Gabriel Piedrahita Uribe, Eduteka, 2007).

El Paso 1 se concentra en la definición del problema de información, se orienta el trabajo individual y en equipos, para plantear la pregunta inicial, analizarla y continuar con la construcción del plan de indagación, esto determina la brújula de búsqueda de información que seguirá el aprendiz. Este paso finaliza cuanso se plantea las preguntas secundarias, a la luz del sistema conceptual de la variable, que representa dicho plan.

El Paso 2 se inicia en base a las preguntas secundarias obtenidas en el Paso 1. En este momento se orienta la importanica de identificar y evaluar las fuentes de información para asegurar su calidad, se utiliza los mejores motores de búsqueda. También se aborda el tema de las normas para las citas de referencia. 
El Paso 3 se dedica al análisis de la información, se parte de los insumos del paso anterior. Se hace un análisis exhaustivo de la información evaluada y seleccionada. Se requiere leer, comprender, comparar y evaluar la información seleccionada. Esta información servirá para responder las preguntas secundarias.

Con las respuestas a las preguntas secundarias se pasa al Paso 4, que implica sistematizar la información y utilizarla. Se realiza un gran esfuerzo de sintesis para responder la pregunta inicial, que orientó todo el proceso de indagación.

Al finalizar el proceso, se elabora un producto final comunicable, que puede ser un ensayo, un organizador gráfico, un artículo, dependiendo de la complejidad y área de estudio. Todo este proceso concluye cuando los estudiantes explican sus hallazgos y muestran sus aprendizajes en los espacios de socialización que el profesor genere para el efecto.

Durante todo el acompañamiento hay retroalimentación permanente, al trabajo individual y en equipo, según la organización diseñada por el docente.

Un detalle importante de resaltar es que el proceso utiliza rúbricas para autoevaluar y coevaluar el proceso en cada paso que el estudiante desarrolla.

\section{Resultados}

La comparación entre los resultados de los tres momentos privilegiados de acceso a la información, esto es, pre-test, post-test1 y post-test final, permitió evidenciar cambios significativos, en los cuatros aspectos fundamentales relacionados con la $\mathrm{CMI}$, a saber: definición del problema de información, búsqueda y evaluación de la información, análisis de la información y en sistematizar la información y utilizarla. La Tabla 1 se refiere al conjunto de involucrados integrados, mientras que la Tabla 2 los caracteriza por campos de formación.

Tabla 1 Tabla de variación de estadísticos de la CMI

\begin{tabular}{|c|c|c|c|}
\hline \multirow{2}{*}{$\begin{array}{c}\text { Estadisticos \% CMI } \\
112 \text { estudiantes }\end{array}$} & \multicolumn{3}{|c|}{ Cursos integrados } \\
\hline & $\begin{array}{c}\text { PRE-TEST } \\
\text { (inicial) }\end{array}$ & $\begin{array}{c}\text { POST-TEST1 } \\
\text { (Primer parcial) }\end{array}$ & $\begin{array}{l}\text { POST-TEST } \\
\text { FINAL }\end{array}$ \\
\hline Promedio & 38,54 & 70,39 & 80,87 \\
\hline Valor minimo & 10,45 & 47,00 & 50,75 \\
\hline Valor máximo & 62,69 & 92,53 & 95,52 \\
\hline Moda & 47,76 & 77.61 & 79,10 \\
\hline Desviación estándar & 11,33 & 10,10 & 7,75 \\
\hline
\end{tabular}


El Modelo Gavilán y la Competencia en manejo de información en la Universidad de las Fuerzas Armadas ESPE

Tabla 2 Tabla de variación de estadísticos de la CMI, por áreas de especialización

\begin{tabular}{|c|c|c|c|}
\hline CMI / Areas especializ. & $\begin{array}{c}\text { ingenierias } \\
\text { técnicas }\end{array}$ & $\begin{array}{c}\text { Ciencias } \\
\text { administrativas }\end{array}$ & $\begin{array}{c}\text { Ciencias de } \\
\text { la educación }\end{array}$ \\
\hline CMI inicial & 43,57 & 40,54 & 31,30 \\
\hline CMI primer parcial & 71,50 & 68,10 & 71,09 \\
\hline CMI final & 81,62 & 80,12 & 80,67 \\
\hline
\end{tabular}

La Figura 5, muestra la evolución de la media aritmética de la CMI estudiantil (conjunto integrado), a lo largo de todo el proceso investigativo.

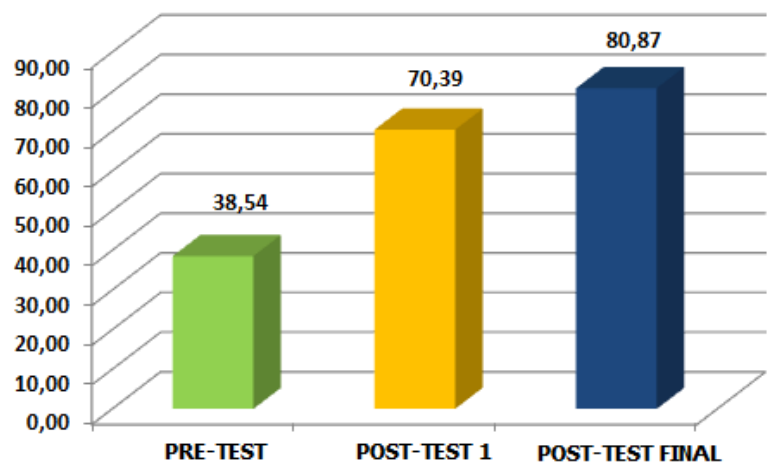

Figura 5 Variación de la media aritmética de la CMI de los estudiantes, por etapas del proceso

Finalmente, la Tabla 3 se relaciona con la Prueba estadística que nos permite sacar conclusiones sobre las hipótesis planteadas.

Tabla 3 Prueba t para medias de dos muestras emparejadas: post-test final y pretest

\begin{tabular}{|c|c|c|}
\hline \multirow[t]{2}{*}{ Prueba t para medias de dos muestras emparejadas } & \multirow[b]{2}{*}{ Variable 1} & \multirow[b]{2}{*}{ Variable 2} \\
\hline & & \\
\hline Media & 80,871964 & 38,542679 \\
\hline Varianala & 60,097135 & 128,36436 \\
\hline Observaciones & 112 & 112 \\
\hline Coeficiente de correlación de Pearson & 0,2224546 & \\
\hline Diferencia hipotética de las medias & 0 & \\
\hline Grados de libertad & 111 & \\
\hline Estadistico $t$ & 36,651951 & \\
\hline P(T<at) uns cola & $3.813 E \cdot 64$ & \\
\hline Valor critico de t (una cola) & 2,3604123 & \\
\hline$P(T<=t)$ des colas & $7,625 E-64$ & \\
\hline Valor critico de $t$ (dos colas) & 2,6208485 & \\
\hline
\end{tabular}


Como se observa en los gráficos, la CMI aumentó considerablemente, en la medida en que los estudiantes adoptaron al Modelo Gavilán como un modelo de búsqueda de información, es decir, el proceso de búsqueda tuvo sentido, porque encontraron un proceso establecido y sobre todo los resultados que cada vez encontraban les fortalecía cada vez más.

\section{Discusión}

En el estudio cuasi-experimental con 112 estudiantes, las pruebas nos permite revisar las dos hipótesis planteadas. Para rechazar la hipótesis nula 1 empleamos el valor de $P$ para dos colas y para rechazar la hipótesis nula 2, utilizamos el valor de $\mathrm{P}$ para una cola. Como se puede apreciar en las dos figuras anteriores, el valor de $\mathrm{P}$, tanto para dos como para una cola es aproximadamente $0 \%$, y es menor al valor alfa $5 \%$, por tanto, las Hipótesis nulas: que el nivel CMI Post-test es menor o igual que el nivel Pre-test es rechazada, así como también que el nivel de CMI Pre-test es mayor o igual que el del Post-test, también es rechazada. Solo nos queda asumir las hipótesis alternativas 1 y 2 , por lo tanto, el nivel de CMI Post-test final es diferente que el nivel de CMI Pre-test, así como el nivel CMI Pre-test es inferior que aquel del Post-test final.

Esto ocurre en los dos momentos del experimento, tanto al comparar el Pre-test con el Post-test 1, luego de seis semanas de los experimentos, como al hacerlo al final del semestre, cinco meses después del inicio del proceso.

Se evidencia un efecto significativo respecto al aumento del nivel de la $\mathrm{CMI}$ en los estudiantes, luego de haber realizado el experimento en los diversos grupos, esto es, la utilización del Modelo Gavilán para desarrollar indagaciones bibliográficas, durante el período de estudio investigado. Estas habilidades para buscar información, seleccionar información, evaluar la información, inciden en el desarrollo de la Competencia en manejo de información de los estudiantes de las dos asignaturas: Metodología de la Investigación y Proyecto Integrador II.

Se puede concluir señalando que los estudiantes cuanto más afirman el uso del Modelo Gavilán, mejoran la calidad de búsquedas y sus resultados de indagación alcanzan niveles de mejor calidad. Por tanto, la competencia para manejar información se consolida y mejora notablemente.

\section{Referencias bibliográficas}

Azinian, H. (2009). La tecnologías de la información y la comunicación en las prácticas pedagógicas: manual apar organizar proyectos. Buenos Aires, Argentina: Centro de publicaciones educativas y material didáctico.

Cacheiro, M. (2014). Educación y tecnología: estrategias didácticas para la integración de las TIC. Madrid, España: Editorial UNED.

Carratalá, F. (2013). La comunicación linguística: como comptencia trasnversal. Madrid, España: Edicione De La Torre. 
El Modelo Gavilán y la Competencia en manejo de información en la Universidad de las Fuerzas Armadas ESPE

Colle, R. (2003). Reflexiones sobre la universidad en la era de la información. Recuperado el 10 de enero de 2015, de Revista Latina de Comunicación Social, 54: http://www.ull.es/publicaciones/latina/200353colle.htm

De Sousa, J. (2000). La Innovación de la Innovación en la Política Los paradigmas emergentes para el desarrollo de estrategias político institucionales. Brasilia.

De Souza, J. (2014). La Innovación de la Innovación en la Política Los paradigmas emergentes para el desarrollo de estrategias político institucionales. Brasilia.

Fundación Gabriel Piedrahita Uribe. (01 de octubre de 2007). Guía para utilizar el Modelo Gavián en el aula. Recuperado el 30 de enero de 2015, de Portal edukativo Eduteka: http://www.eduteka.org/pdfdir/GuiaGavilan.pdf

Galindo, J. (3 de junio de 1996). Cultura de la información, política y mundos posibles. Recuperado el 17 de enero de 2015, de Revista Estudios sobre las culturas contemporáneas, vol. 2, núm. 3: <http://www.redalyc.org/articulo.oa?id=31600302

Garmendia, L. (2005). La alfabetización informacional como estímulo investigativo: una estrategia en la gestión de la información y el conocimiento. Recuperado el 23 de enero de 2015, de Biblios: Revista electrónica de bibliotecología, archivología y museología, №. 21.

González-Fernández-Villavicencio, N. (2 de octubre de 2014). ALFIN 2.0: Herramientas 2.0 en programas ALFIN en bibliotecas de la Universidad de Sevilla. Recuperado el 30 de enero de 2015, de e-LiS: http://eprints.rclis.org/14140/

Juárez-López, S., \& López-Pérez, J. (2012). El Método Arenas: Aplicación del PBL para la formación de científicos en países con economías en desarrollo. Bloomington, EE.UU.: Palibrio.

Moya, J., \& Luengo, F. (2011). Teoría y práctica de las competencias básicas. Barcelona: Editorial GRAÓ.

Sánchez, N., \& Alfonso, I. (2007). Las competencias informacionales en las ciencias biomédicas: una aproximación a partir de la literatura publicada. Recuperado el 21 de febrero de 2015, de ACIMED, vol. 15, n. 2.: http://eprints.rclis.org/9097/

Silva, J. D. (2014). La Innovación de la Innovación en la Política Los paradigmas emergentes para el desarrollo de estrategias político institucionales. Brasilia.

Vivancos, J. (2008). Tratamiento de la información y competencia digital. Madrid, España: Alianza Editorial S.A. 